\title{
On Piezoelectric Energy Harvesting from Human Motion
}

\author{
Chunhua Sun*, Guangqing Shang, Hongbing Wang \\ Department of Mechanical and Electrical Engineering, Suzhou Vocational University, Suzhou, China \\ Email: *chh_sunny@163.com
}

How to cite this paper: Sun, C.H., Shang, G.Q. and Wang, H.B. (2019) On Piezoelectric Energy Harvesting from Human Motion. Journal of Power and Energy Engineering, 7, 155-164.

https://doi.org/10.4236/jpee.2019.71008

Received: December 30, 2018

Accepted: January 27, 2019

Published: January 30, 2019

Copyright $\odot 2019$ by author(s) and Scientific Research Publishing Inc. This work is licensed under the Creative Commons Attribution International License (CC BY 4.0).

http://creativecommons.org/licenses/by/4.0/

(c) (i) Open Access

\begin{abstract}
With the rapid development of low-power communication technology and microelectronics technology, wearable and portable embedded health monitoring devices, micro-sensors, and human body network positioning devices have begun to appear. For seeking reliable energy sources to replace battery on these devices, it is of great significance for developing low power products to explore the research of piezoelectric effect in conversion of human motion into electricity. Based on the different human motions, the existing technology of piezoelectric energy harvester (PEH) is firstly classified, including PEHs through heel-strike, knee-joint, arm motion, center of mass. The technology is then summarized and the direction of future development and efforts is further pointed out.
\end{abstract}

\section{Keywords}

Biochemical Energy, Piezoelectric Effect, Piezoelectric Energy Harvester (PEH)

\section{Introduction}

In today's environmental friendly society, it is increasingly urgent to replace chemical batteries with biomechanical energy. With the development of low power products such as the portable electric devices, GPS and MEMS, whose power is low to milliwatts, biomechanical energy harvesting from human motion presents a promising clean alternative [1].

Biomechanical energy harvesters generate electricity from people as they go about their activities of daily living resulting in power generation over much longer durations [2]. The conversion modes of biomechanical energy from human motion are mainly electromagnetic, mechanical, thermoelectric and piezoelectric [3]. Among these, the piezoelectric mode has become one focus in that its simple structure, pollution-free, high power density and so on. There have 
been three main ways to collect human motion energy with piezoelectric effect: bending piezoelectric material to produce electricity by using the weight of human body; causing vibration of piezoelectric beam by using acceleration pulse generated when heel collision with the ground or leg swinging. Besides, gripping palm and expanding arm's muscles can also be used to generate electricity from piezoelectric material [4]. The objective of this paper is thus to review the currently available piezoelectric energy harvester from different forms of human motion, then to summary the technology. The main challenges that must be overcome to reach this goal are finally discussed.

\section{PEHs from Human Motions}

\section{Energy Harvesting from Walking}

Based on walking, the PEHs were triggered through heel-strike, knee-joint, arm motion, center of mass. The developed PEH devices mainly included shoe, kneepad, floor, dancing blanket, and backpack.

\section{PEHs through heel strike}

Kymissis and et al. [5] proposed three different devices that could be built into a shoe and generated electrical power parasitically while walking, as shown in Figure 1. The devices included a "Thunder" actuator constructed of piezoceramic composite material located in the heel, a rotary magnetic generator also located under the heel, and a multilayer PVDF foil laminate patch located in the sole of the shoe. In order to compare the performance of the three devices, three working prototypes were constructed and their performances were measured. The peak powers were observed to approach $20 \mathrm{~mW}$ for the PVDF stave, $80 \mathrm{~mW}$ for the PZT unimorph, and the shoe mounted rotary generator averaged to about $250 \mathrm{~mW}$. A follow-up energy storage circuit and a radio frequency signal transmitting device were also designed. The energy generated by walking $3-6$ steps per second could transmit a radio frequency signal. This design replaced batteries to supply power for GPS positioners, walkthroughs and other devices.



Figure 1. PEH on shoe proposed by Kymissis and et al. [5]. 
On this basis, further research had been done on structural optimization design and experimental verification by Fourie [6] and Mateu [7].

Haghbin [8] made a PEH with an air pump, as shown in Figure 2(a). PZT films were deformed by compressed air, which generated electrical energy. The whole PEH device was sealed to protect the piezoelectric material by airbag. It was very convenient to implant soles and generate electricity by treading on heels, as shown in Figure 2(b). Experiments on a running machine showed that the average power of $1.24 \mathrm{~mW}$ could be generated at a walking speed of 4 miles/h.

Besides, Leinonen and et al. [9] designed a cymbal PEH and implanted it into the sole, as shown in Figure 3. PZT-5H disc with $\Phi 35 \mathrm{~mm}$ was selected to stamp into "cymbal" structure, which produced deformation under the weight of human body. The experimental results showed that the maximum average power of about $800 \mathrm{uW}$ could be generated at $1 \mathrm{~Hz}$ gait. The theoretical and experimental errors were only within $7 \%$.

Li and et al. [10] firstly designed a PEH with a curve L-shape mass at free end of the piezoelectric cantilever beam from the point of view of improving the energy collection density and reducing the resonant frequency. The piezoelectric beam was mounted in a cavity and embedded in sole, as shown in Figure 4. The power density could reach $1.45 \mathrm{~mW} / \mathrm{cm}^{3}$, which was about $68 \%$ higher than that of traditional piezoelectric cantilever beam. The experimental results showed

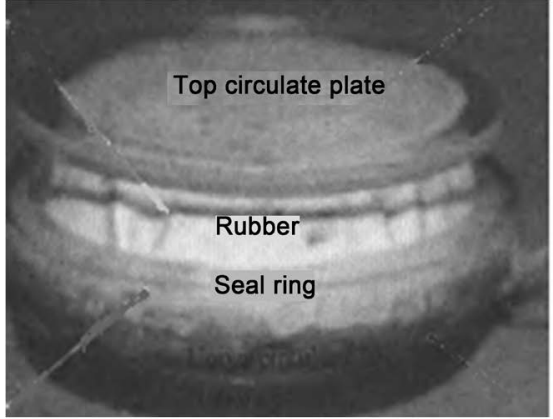

(a)

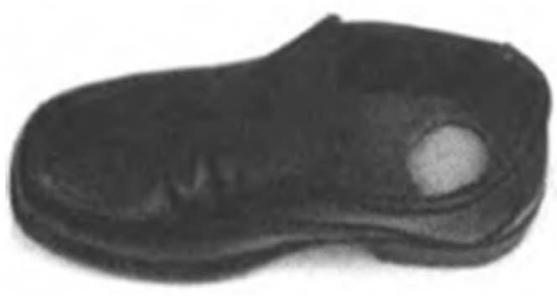

(b)

Figure 2. PEH proposed by Haghbin [8]. (a) Air pump type PEH; (b) Inserted in shoe

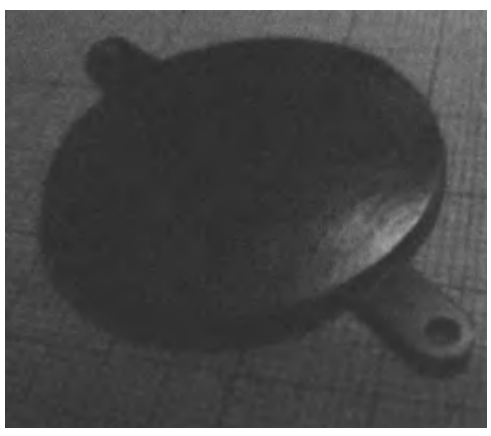

(a)

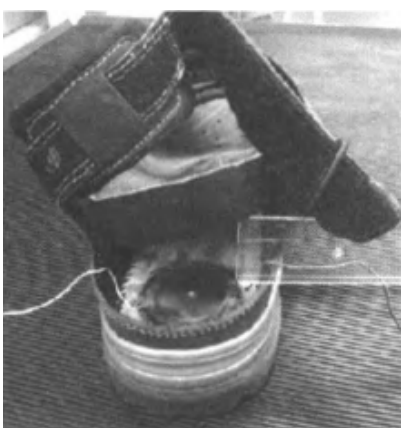

(b)

Figure 3. Piezoelectric energy harvesting shoe proposed by Leinonen [9]. (a) Cymbal $\mathrm{PEH}$; (b) Inserted in shoe. 
that the average output power was $49 \mathrm{uW}$ at a walking speed of 3 miles per hour.

Yoshiyasu Takefuji et al. [11] developed a piezoelectric carpet, which was tested at the entrance of the subway or the places where there are more pedestrians in the corridors of shopping malls. The results showed that the $25 \mathrm{~m}^{2}$ of the developed carpet produced $1400 \mathrm{~kW}$ of electricity per day. Japan's East Japan Raiway also installed the "power generation mat" for harvesting energy from pedestrian to power the "ticket automatic door", as shown in Figure 5.

The piezoelectric power generated floor was exhibited on Shanghai Science and Technology Festival of 2011, as shown in Figure 6. The floor was developed by Shanghai Silicate Research Institute of the Chinese Academy of Sciences. When pedestrians were jumping or walking on this floor, it could instantly generate electricity to light up the LED lattice and display the harvested energy data on the large screen [12].

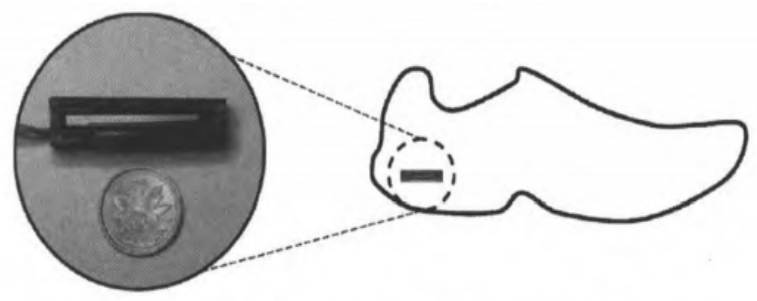

Figure 4. Piezoelectric energy harvesting shoe proposed LI and et al. [10].
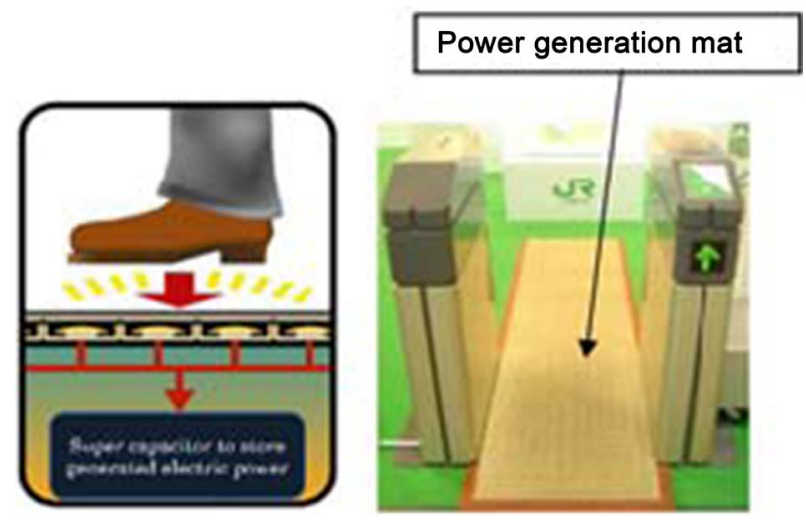

Figure 5. Power generation mat in Japan [11].

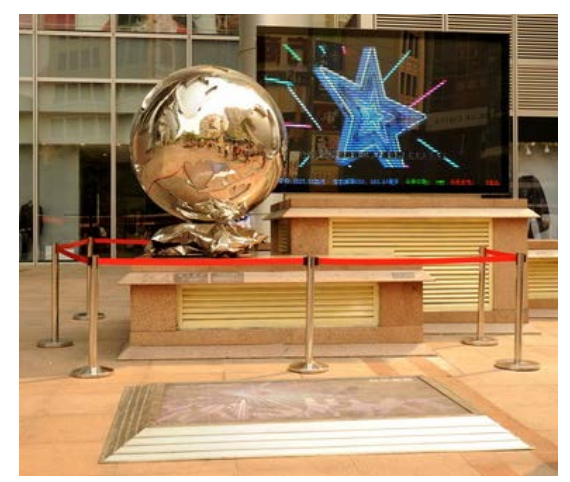

Figure 6. Piezoelectric power generated floor [12]. 
Hwang et al. [13] designed a pedal-type piezoelectric vibration energy collection device, as shown in Figure 7. The experimental results show that when a person of $68 \mathrm{~kg}$ walked on the tiles at both ends of the device, four piezoelectric beams was dived by the spring connected with the tiles for vibrating, an average power of about $0.12 \mathrm{~mW}$ in a cycle was generated. When a $80 \mathrm{~g}$ steel ball fell from $1 \mathrm{~m}$ height to the developed ceramic tile, the average power of about 707 uW could be generated.

Because the resonance frequency band of the traditional linear piezoelectric cantilever beam is too narrow, many researchers have begun to introduce non-linearity into the field of piezoelectric vibration energy harvesting from human motion. Wang Wei et al. [14] researched effect of output power on different harvesters, including bi-stable energy harvester $(\mathrm{BEH})$, linear energy harvester (LEH) and mono-stable energy harvester (MEH), as shown in Figure 8. They designed a bistable magnetically coupled piezoelectric cantilever beam for harvesting human motion. The potential well was obtained by acceleration through leg swing and the foot impact. The energy capture efficiency improved very much.

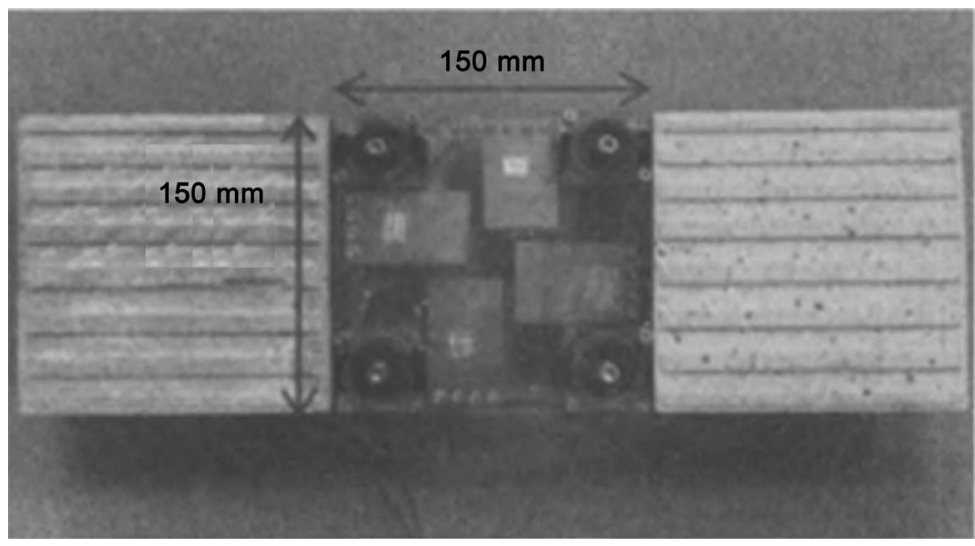

Figure 7. A pedal-type $\mathrm{PEH}$ device [13].

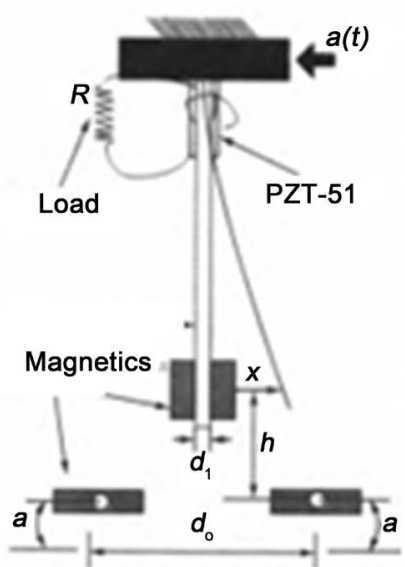

(a)

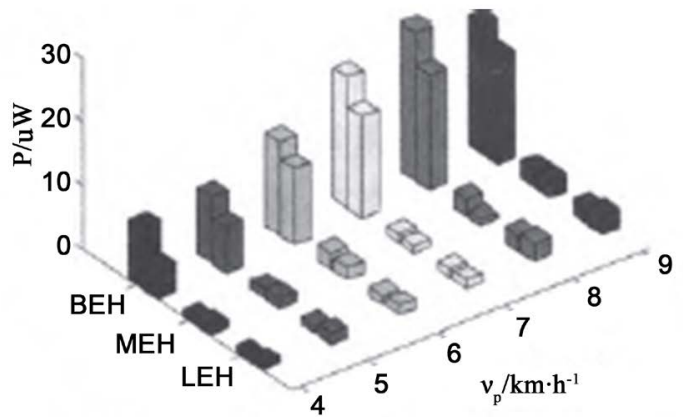

(b)

Figure 8. Non-linear PEH device proposed by Wang et al. [14]. (a) Non-linear PEH device; (b) Output power via different kinds of harvesters. 


\section{PEHs through knee joint}

PEH device through knee joint was developed by Donelan and et al. in 2008 [15]. This $1.6 \mathrm{~kg}$ device comprised an orthopedic knee brace configured such that knee motion drove a gear train (113:1) through a unidirectional clutch, transmitting only knee extension motion to a DC brushless motor that served as the generator. The generated electrical power was dissipated by a load resistor. This method generated $2.5 \mathrm{~W}$ per knee at a walking speed of $1.5 \mathrm{~m} / \mathrm{s}$.

To improve working stability and comfortability, Yao et al. [16] subsequently proposed a harvester installed on keen, as shown in Figure 9(a). The device adopted a mechanism of cam and roller (in Figure 9(b)) to transfer leg movement to piezoelectric ceramics, which generated electrical energy in each step cycle. In the experiment, the piezoelectric harvester with $\Phi 40 \mathrm{~mm}$ substrate and $\Phi 30 \mathrm{~mm}$ piezoelectric ceramic wafer was selected. When $100 \mathrm{k} \Omega$ was applied, the maximum output voltage was $80 \mathrm{~V}$ and the maximum output power was $58.2 \mathrm{~mW}$, and about $27.5 \mathrm{~mJ}$ of energy could be generated per walk.

Pozzi and et al. [17] designed a PEH (shown in Figure 10) by using the leg swing motion as the power source. It consisted of four parts: rotor, stator, dial and piezoelectric beam. The PEH was installed near the knee joint. When the leg swings back and forth, the piezoelectric cantilever beam was continuously



(a)

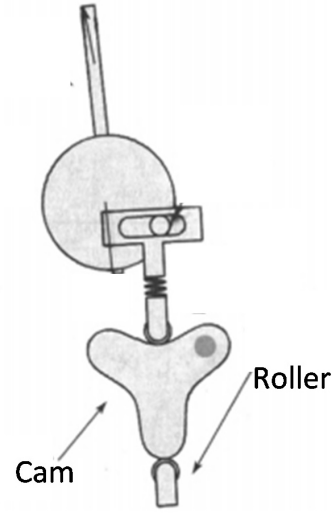

(b)

Figure 9. A PEH proposed by Yao and et al. [16]. (a) Setup installed on keen; (b) Mechanism of cam and roller.


Figure 10. A PEH proposed by Pozzi and et al. [17] 
picked by the dial to generate electricity. The experimental result showed that up to $2.06 \mathrm{~mW}$ of power was generated while a man weared the device on leg and carried a $24 \mathrm{~kg}$ backpack.

\section{PEHs through arm motion}

Bomm Yang and et al. [18] made full use of the soft properties of PVDF materials and proposed an effective shell structure for energy harvesting from twist joint. PVDF polymer film was attached on the curved shell structure, which was used to protect twist joint, as shown in Figure 11. The experiment validated that the output voltage of the shell structure was higher than that of the flat one. When the angular velocity of elbow joint was $9 \mathrm{rad} / \mathrm{s}$, the maximum voltage was up to $40 \mathrm{~V}$.

Renaud and et al. [19] developed a hand-crashed piezoelectric energy collector, as shown in Figure 12. A piezoelectric cantilever beam was installed at both ends of a cavity, and a track which accommodated the reciprocating motion of the slider was designed in the vertical direction. The experimental results showed that the slider with a mass of about $4 \mathrm{~g}$ generated electricity about $600 \mathrm{uW}$ when it collided with the piezoelectric beams on both sides of the track at $10 \mathrm{~Hz}$.

Similarly, Halim proposes a hand-operated vibration energy harvester with hybrid piezoelectric and electromagnetic power generation modes [20]. The piezoelectric beam impacted by ball was driven to generate high frequency resonance. Meanwhile the fixed magnet on the piezoelectric beam moved relative to the coil, so that the piezoelectric and electromagnetic power generation could be realized simultaneously. Compared with the same type of electromagnetic power generation device, the power density was greatly increased.

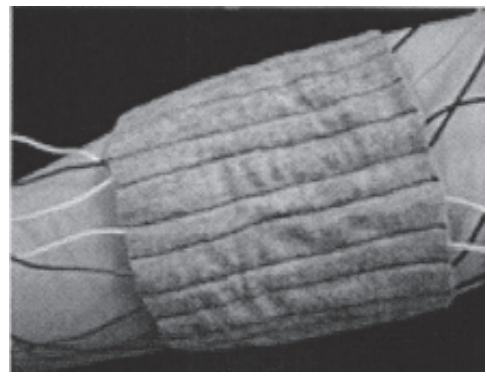

Figure 11. Flexible PEH by Momm Yallg and et al. [18].

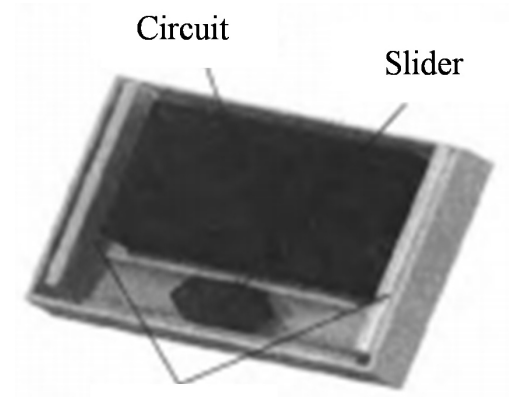

Piezoelectric beam

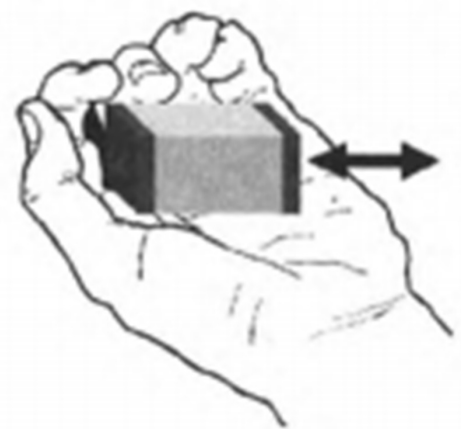

Figure 12. Hand-crashed PEH proposed by Renaud and et al. [19]. 


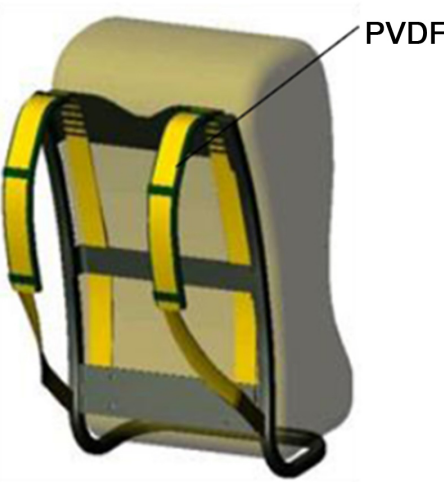

(a)

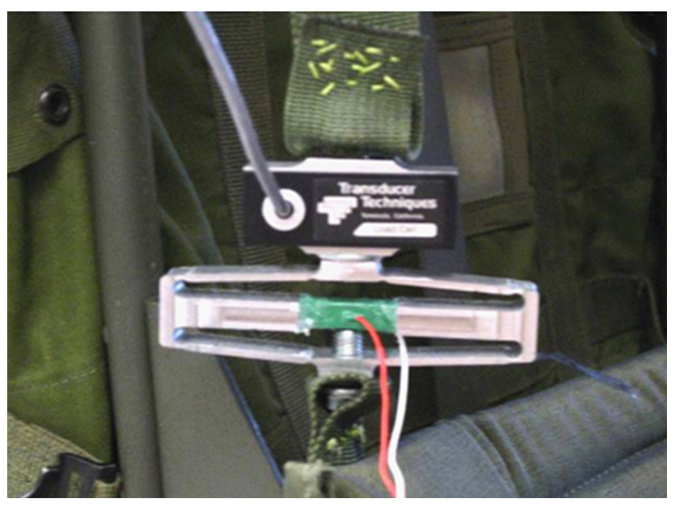

(b)

Figure 13. Piezoelectric energy harvesting backpack proposed Joel and et al. [22]. (a) Piezoelectric backpack; (b) the stack amplifier.

\section{PEHs through backpack with center of mass}

Granstrom and et al. [21] took full advantage of the flexible properties of PVDF piezoelectric materials and installed PVDF in the backstrap of shoulder bags. PVDF could be strained by the weight of the backpack to generate electricity. Experiment showed that the average power of $45.6 \mathrm{~mW}$ was generated. Obviously, the flexible material using PVDF was easier to excite piezoelectric effect for capturing more energy than hard ceramics such as piezoelectric stack.

To collect more energy from backpack, Joel and et al. [22] proposed a piezoelectric energy harvesting backpack, as shown in Figure 13(a). It was accomplished by replacing the strap buckle with a mechanically amplified piezoelectric stack actuator (as shown in Figure 13(b)). The instrument allowed the relatively low forces generated by the pack to be transformed to high forces on the piezoelectric stack. Using the instrumented backpack carrying a $220 \mathrm{~N}$ load at $\sim 2.75$ $\mathrm{Hz}$, the mean power output of $\sim 0.4 \mathrm{~mW}$ could be available from each piezoelectric device. Because piezoelectric stack was used and the material was too hard to deform, the effect of energy collection was not very good.

\section{Summary}

The technology of piezoelectric energy harvesting from walking, knee or muscle motion, and palm grasping, has been researched. The developed PEHs were applied in shoe, backpack, and wrist protector. The harvested electricity was generally at the milliwatt level. It was mainly used in low power electronic equipment for power supplying. However, there exists a long way to go for practical application. Some problems must be overcome in the future research including lightweight, flexibility, stretchable, multi-direction and wideband, to be more fit to human body structure and movement.

\section{Conflicts of Interest}

The authors declare no conflicts of interest regarding the publication of this paper. 


\section{References}

[1] Li, Q.G., Naing, V. and Donelan, M.J. (2009) Development of a Biomechanical Energy Harvester. Journal of NeuroEngineering and Rehabilitation, 6, 22. https://doi.org/10.1186/1743-0003-6-22

[2] Starner, T. and Paradiso, J.A. (2005) Human Generated Power for Mobile Electronics. In: Piguet, C., Ed., Low-Power Electronics Design, CRC Press, Boca Raton, 1-30.

[3] Riemer, R. and Shapiro, A. (2011) Biomechanical Energy Harvesting from Human Motion: Theory, State of the Art, Design Guidelines, and Future Directions. Journal of NeuroEngineering and Rehabilitation, 8, 22.

http://www.jneuroengrehab.com/content/8/1/22 https://doi.org/10.1186/1743-0003-8-22

[4] Wei, S. and Hu, H. (2018) A Review of Human Motion Energy Harvesting Based on Piezoelectric Vibration. Machinery \& Electronics, 36, 67-72.

[5] Kymissis, J., Kendall, C., Paradiso, J. and Gershenfeld, N. (1998) Parasitic Power Harvesting in Shoes. Second International Symposium on Wearable Computers, Pittsburg, PA, 19-20 October 1998, 132-139.

[6] Fourie, D. (2010) Shoe-Mounted PVDF Piezoelectric Transducer for Energy Harvesting. MITUG Research Journal, 19, 66-70.

[7] Mateu, L. and Moll, F. (2005) Optimum Piezoelectric Bending Beam Structures for Energy Harvesting Using Shoe Inserts. Journal of Intelligent Material Systems and Structures, 16, 835-845. https://doi.org/10.1177/1045389X05055280

[8] Haghbin, N. (2011) Shoe Embedded Air Pump Type Piezoelectric Power Harvester. Ryerson University, Toronto.

[9] Leinonen, M., Palosssri, J., Juuti, J., et al. (2013) Combined Electrical and Electromechanical Simulations of a Piezoelectric Cymbal Harvester for Energy Harvesting from Walking. Journal of Intelligent Material Systems and Structures, 25, 391-400.

[10] Li, W.G., He, S. and Yu, S. (2010) Improving Power Density of a Cantilever Piezoelectric Power Harvester through a Curved L-Shaped Proof Mass. IEEE Transactions on Industrial Electronics, 57, 868-876. https://doi.org/10.1109/TIE.2009.2030761

[11] Takefuji, Y. (2008) Known and Unknown Phenomena of Nonlinear Behaviors in the Power Harvesting Mat and the Wave Speaker. Keio University, Fujisawa.

[12] The "Piezoelectric Generation Floor" Is Displayed on the Pedestrian Street of Nanjing Road. http://www.sic.ac.cn/zt/kpwsx/kpxw/201105/t20110519_3136905.html

[13] Hwang, S.J., Jung, H.J., Kim, J.H., et al. (2015) Designing and Manufacturing a Piezoelectric Tile for Harvesting Energy from Footsteps. Current Applied Physics, 15, 669-674. https://doi.org/10.1016/j.cap.2015.02.009

[14] Wang, W., Cao, J.Y., Lin, J., Zhou, S.X. and Cai, Y.L. (2015) Nonlinear Bi-Stable Energy Harvester from Human Motion. Journal of Xi an Jiaotong University, 49, 58-63.

[15] Donelan, J.M., Li, Q., Naing, V., Hoffer, J.A., Weber, D.J. and Kuo, A.D. (2008) Biomechanical Energy Harvesting: Generating Electricity during Walking with Minimal User Effort. Science, 319, 807-810. https://doi.org/10.1126/science.1149860

[16] Yao, Y. and Yao, M. (2011) Design and Experimental Study of a Human-Body Energy Conversion System Based on Piezoelectric Ceramic. Machine Design and Research, 27, 34-37.

[17] Pozzi, M., Min, S.H.A., Zhu, M., et al. (2012) The Pizzicato Knee-Joint Energy Harvester: Characterization with Biomechanical Data and the Effect of Backpack Load. Smart Materials and Structures, 21, 75023-75030. 
https://doi.org/10.1088/0964-1726/21/7/075023

[18] Yang, B. and Yun, K.S. (2011) Efficient Energy Harvesting from Human Motion Using Wearable Piezoelectric Shell Structures. Solid-State Sensors, Actuators and Microsystems Conference, 5-9 June 2011, 2646-2649.

[19] Renaud, M., Fiorini, P., Schaijk, R.V., et al. (2009) Harvesting Energy from the Motion of Human Limbs: The Design and Analysis of an Impact-Based Piezoelectric Generator. Smart Materials and Structures, 18, 35001. https://doi.org/10.1088/0964-1726/18/3/035001

[20] Halim, M.A. and Park, J.Y. (2015) Modeling and Experiment of a Handy Motion Driven, Frequency Up-Converting Electromagnetic Energy Harvester Using Transverse Impact by Spherical Ball. Sensors and Actuators A: Physical, 229, 50-58. https://doi.org/10.1016/j.sna.2015.03.024

[21] Granstrom, J., Feenstra, J., Sodano, H.A., et al. (2007) Energy Harvesting from a Backpack Instrumented with Piezoelectric Shoulder Straps. Smart Materials and Structures, 16, 1810-1820. https://doi.org/10.1088/0964-1726/16/5/036

[22] Feenstra, J., Granstrom, J. and Sodano, H. (2008) Energy Harvesting through a Backpack Employing a Mechanically Amplified Piezoelectric Stack. Mechanical Systems and Signal Processing, 22, 721-734.

https://doi.org/10.1016/j.ymssp.2007.09.015 